OPEN ACCESS

Edited by:

Jinyi Lin,

Nanjing Tech University, China

Reviewed by:

Xiaobao Xu,

Nanjing University of Science and

Technology, China

Xuewen Wang,

Northwestern Polytechnical

University, China

*Correspondence:

Caihou Lin

lincai76@163.com

Qiushui Chen

qchen@fzu.edu.cn

Huanghao Yang

hhyang@fzu.edu.cn

Specialty section:

This article was submitted to Physical Chemistry and Chemical

Physics,

a section of the journal

Frontiers in Chemistry

Received: 17 March 2021

Accepted: 26 March 2021

Published: 26 April 2021

Citation:

Xie L, Zan J, Yang Z, Wu Q, Chen X, Ou X, Lin C, Chen $Q$ and Yang $H$ (2021) A Perovskite-Based Paper Microfluidic Sensor for Haloalkane Assays. Front. Chem. 9:682006. doi: 10.3389/fchem.2021.682006

\section{A Perovskite-Based Paper Microfluidic Sensor for Haloalkane Assays}

\author{
Lili Xie ${ }^{1}$, Jie Zan ${ }^{1}$, Zhijian Yang ${ }^{1}$, Qinxia Wu ${ }^{1}$, Xiaofeng Chen ${ }^{1}$, Xiangyu Ou ${ }^{1}$, Caihou Lin ${ }^{2 *}$, \\ Qiushui Chen ${ }^{1,3 *}$ and Huanghao Yang ${ }^{1,3 *}$ \\ ${ }^{1}$ Ministry of Education (MOE) Key Laboratory for Analytical Science of Food Safety and Biology, Fujian Provincial Key \\ Laboratory of Analysis and Detection Technology for Food Safety, College of Chemistry, Fuzhou University, Fuzhou, China, \\ ${ }^{2}$ Department of Neurosurgery, Fujian Medical University Union Hospital, Fuzhou, China, ${ }^{3}$ Fujian Science and Technology \\ Innovation Laboratory for Optoelectronic Information of China, Fuzhou, China
}

Detection of haloalkanes is of great industrial and scientific importance because some haloalkanes are found serious biological and atmospheric issues. The development of a flexible, wearable sensing device for haloalkane assays is highly desired. Here, we develop a paper-based microfluidic sensor to achieve low-cost, high-throughput, and convenient detection of haloalkanes using perovskite nanocrystals as a nanoprobe through anion exchanging. We demonstrate that the $\mathrm{CsPbX}_{3}(X=\mathrm{Cl}, \mathrm{Br}$, or I) nanocrystals are selectively and sensitively in response to haloalkanes $\left(\mathrm{CH}_{2} \mathrm{Cl}_{2}, \mathrm{CH}_{2} \mathrm{Br}_{2}\right)$, and their concentrations can be determined as a function of photoluminescence spectral shifts of perovskite nanocrystals. In particular, an addition of nucleophilic trialkyl phosphines (TOP) or a UV-photon-induced electron transfer from $\mathrm{CsPbX}_{3}$ nanocrystals is responsible for achieving fast sensing of haloalkanes. We further fabricate a paper-based multichannel microfluidic sensor to implement fast colorimetric assays of $\mathrm{CH}_{2} \mathrm{Cl}_{2}$ and $\mathrm{CH}_{2} \mathrm{Br}_{2}$. We also demonstrate a direct experimental observation on chemical kinetics of anion exchanging in lead-halide perovskite nanocrystals using a slow solvent diffusion strategy. Our studies may offer an opportunity to develop flexible, wearable microfluidic sensors for haloalkane sensing, and advance the in-depth fundamental understanding of the physical origin of anion-exchanged nanocrystals.

Keywords: colorimetric, microfluidic, anion exchange, perovskite, haloalkanes

Haloalkanes are an important group of chemical compounds widely used as solvents and reactants in pharmaceutical and chemical industries (Kinani et al., 2016; Daud et al., 2018). Accurate detection and identification of haloalkanes are of great industrial and scientific importance (Leri et al., 2006; Fu et al., 2020; Gul et al., 2020), because many of these compounds exhibit high toxicities to environment and human health, such as carcinogenicity and nephrotoxicity. Over the years, several techniques have been developed for the detection of haloalkanes, such as chromatic, fluorescent indicators, X-ray absorption near-edge structure (XANES) spectroscopy, ultrahighresolution mass spectrometry (UHR-MS), and liquid chromatography-mass (LC-MS) (Leri et al., 2006; Roveretto et al., 2019; Fu et al., 2020; Gul et al., 2020; Li et al., 2020; Yin et al., 2021). 
Despite its importance, precise and rapid quantification of haloalkanes remains a technical challenge. Inherent limitations, such as the bandwidth of recording, restrict the available resolution. Additionally, these technologies are generally limited by their lack of high selectivity. For practical applications, rapid and high-throughput analysis of pollutants are highly desired for on-site testing.

Lead-halide perovskite nanocrystals (NCs) are an emerging class of materials that could be used to achieve fast, sensitive, and selective detection of halides through anion exchange, owing to its unique property of soft and predominantly ionic lattice (Akkerman et al., 2018; Chen et al., 2018; Geng et al., 2018). These materials have been well-developed to be applied in solar cells, light-emitting devices, photodetectors, and photocatalysis, because of their unique facile synthesis, high photoluminescence quantum yields and optical versatility (Huang et al., 2016; Zhou et al., 2016; Kovalenko et al., 2017; Li et al., 2017; Yin et al., 2017). Multicolor photoluminescence emissions can be readily tuned to various wavelengths in the visible spectrum either by adjusting the ratio of halide atoms $(\mathrm{Cl}, \mathrm{Br}, \mathrm{I})$ or by facile anion exchange (Xing et al., 2014; Protesescu et al., 2015; Wong et al., 2019). Notably, the high mobility of halide anions and the high concentration of halide vacancies result in fast rate during anion exchanging (Parobek et al., 2017; Yoon et al., 2018; Zheng et al., 2018). Direct observation of dynamic process in anion exchanging is still difficult, owing to its fast chemical kinetics, typically within a few seconds (Pan et al., 2018). Although leadhalide perovskite nanocrystals are promising in haloalkanes sensing (Zhu Y. et al., 2019; Li et al., 2021), the fabrication of an on-site testing device is highly desired for low-cost, convenient applications.

Over the past two decades, microfluidic lab-on-a-chip (LOC) technologies have increasingly emerged as a powerful tool for point of care testing, by taking the advantages of low sample consumption, low-cost production, and high-throughput rapid analysis (Xie et al., 2019; Miller et al., 2020). In particular, paperbased microfluidic sensors are attractive to perform the realtime measurements in-situ by designing an on-demand pattern of the channels (Cate et al., 2015). This makes paper-based microfluidic devices of particular interest in testing analyses when combined with luminescence nanocrystals. Here, we demonstrate a paper-based multichannel microfluidic platform for detection of haloalkanes through anion exchanging in

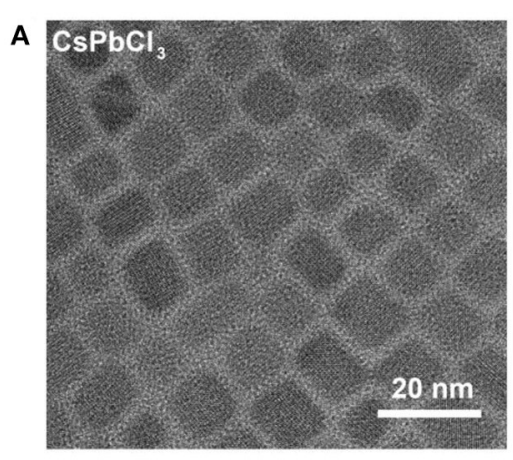

B

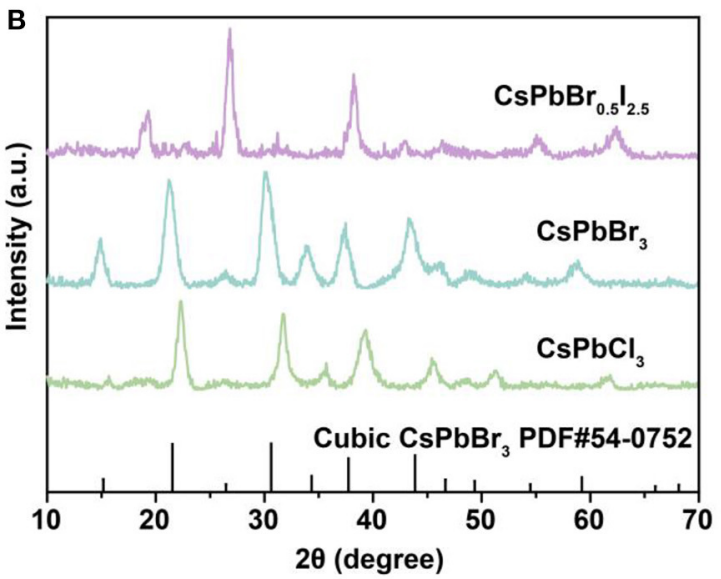

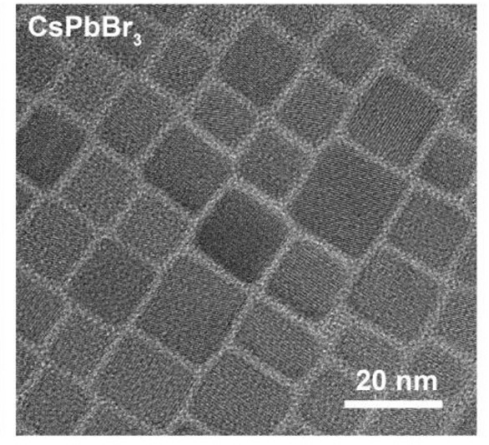
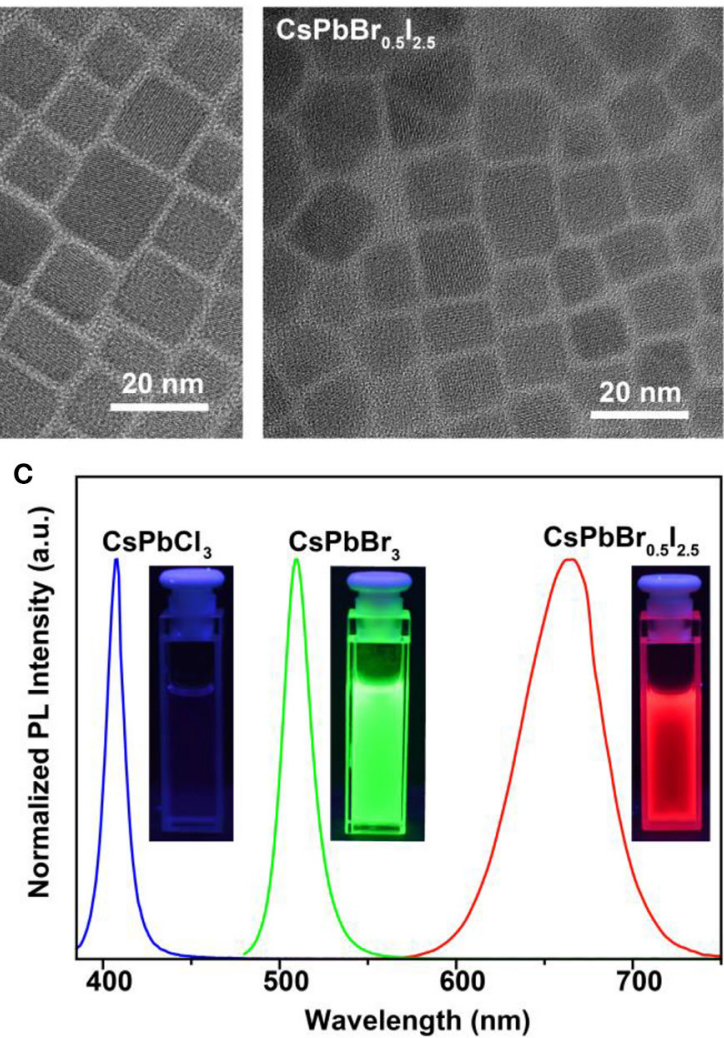

FIGURE 1 | Characterization and spectroscopy study of as-synthesized $\mathrm{CsPbX}_{3}(\mathbf{X}=\mathrm{Cl}$, $\mathrm{Br}$, or I) perovskite nanocrystals. (A) TEM images of the as-synthesized cubic-phase perovskite nanocrystals. The samples are $\mathrm{CsPbCl}_{3}, \mathrm{CsPbBr}_{3}$, and $\mathrm{CsPbBr}_{0.5} \mathrm{I}_{2.5}$ nanocrystals. (B) XRD patterns of the as-synthesized perovskite nanocrystals. All peaks are consistent with the cubic-phase CsPbBr3 structure [Joint Committee on Powder Diffraction Standards file (PDF) number 54-0752]. (C) Fluorescence spectra of the perovskite nanocrystals under 365-nm UV excitation. The insets show photographs of the samples under 365-nm UV excitation. 
perovskite nanocrystals. We show a direct observation of the chemical kinetics of anion exchanging between perovskite nanocrystals and haloalkanes, as a result of a slow solvent diffusion. We also demonstrate that the lead-halide perovskite nanocrystals-based paper microfluidic sensor is affordable to achieve a fast, convenient analysis of haloalkanes based on colorimetric sensing.

To validate our hypothesis, we synthesized lead-halide perovskite nanocrystals by reacting Cs-oleate precursors with $\mathrm{PbX}_{2}(\mathrm{X}=\mathrm{Cl}, \mathrm{Br}$ or $\mathrm{I})$, using a hot-injection solution strategy at $160^{\circ} \mathrm{C}$. Transmission electron microscopy (TEM) images indicate the well-defined cubic morphologies of the assynthesized $\mathrm{CsPbCl}_{3}, \mathrm{CsPbr}_{3}$, and $\mathrm{CsPbBr}_{0.5} / \mathrm{I}_{2.5}$ perovskite nanocrystals (Figure 1A), with an average size of about $11 \mathrm{~nm}$ (Supplementary Figure 1). X-ray diffraction (XRD) measurements were conducted to confirm the cubic phase of the perovskite nanocrystals (Figure 1B). Figure 1C shows photoluminescence spectra of the as-synthesized perovskite nanocrystals, which display high photoluminescence yields and color-tunable emissions in blue, green, and red regions. The highly ionic nature of perovskite nanocrystals allows for readily engineering their optical emissions through facile and rapid anion exchange. Such unique capability can be employed to achieve rapid detection of halide compounds through anion exchanging.

To assess the feasibility of the perovskite nanocrystals as nanoprobes for colorimetric sensing of haloalkanes, we used $\mathrm{CH}_{2} \mathrm{Br}_{2}$ as an analyte sample by reacting with
$\mathrm{CsPbCl}_{3}$ and $\mathrm{CsPbBr}_{0.5} \mathrm{I}_{2.5}$ nanocrystals. Our experimental results indicated that their luminescence emission colors were readily shifted as a result of the anion exchanging process (Figure 2A; Supplementary Figures 2, 3). Note that the passivation of $\mathrm{CsPBr}_{3}$ nanocrystals with bromideenriched $\mathrm{CH}_{2} \mathrm{Br}_{2}$ molecules can enhance their luminescence emission, owing to the efficiently reduced surface quenching defects (Supplementary Figure 4). Moreover, the use of TOP or UV illumination is capable of accumulating the anion exchanging rates (Supplementary Figures 5, 6). The wavelength shift in the photoluminescence emission spectra is attributed to the change of bandgaps of the perovskite nanocrystals as a result of the exchange of bromide with $\mathrm{Cl}$ or $\mathrm{I}$ in the lattice of $\mathrm{CsPbBr}_{3}$ and $\mathrm{CsPbBr}_{0.5} \mathrm{I}_{2.5}$ nanocrystals. The changes in emission color are conveniently visual for colorimetric sensing of samples, as indicated in the CIE chart (Figure 2B). Furthermore, we examined the suitability of using this method to achieve a quantitative detection of $\mathrm{CH}_{2} \mathrm{Cl}_{2}$ and $\mathrm{CH}_{2} \mathrm{Br}_{2}$ samples through measuring the wavelength shift of photoluminescence from perovskite nanocrystals, as shown in Figures $\mathbf{2 C , E}$. With the increase in the analyte concentrations, the photoluminescence emission wavelength of the $\mathrm{CsPbBr}_{3}$ nanocrystals in solution was shifted from 510 to $460 \mathrm{~nm}$, with a linear equation of $y=0.69 x+510.2$ ( $\mathrm{y}$ is the wavelength and $\mathrm{x}$ is the sample concentration) and a detection limit of $4.12 \mathrm{mg} / \mathrm{ml}$ for $\mathrm{CH}_{2} \mathrm{Cl}_{2}$. Similarly, we demonstrated a detection limit of $0.29 \mathrm{mg} / \mathrm{ml}$ for $\mathrm{CH}_{2} \mathrm{Br}_{2}$ sensing (Figures 2D,F). These results suggested that our

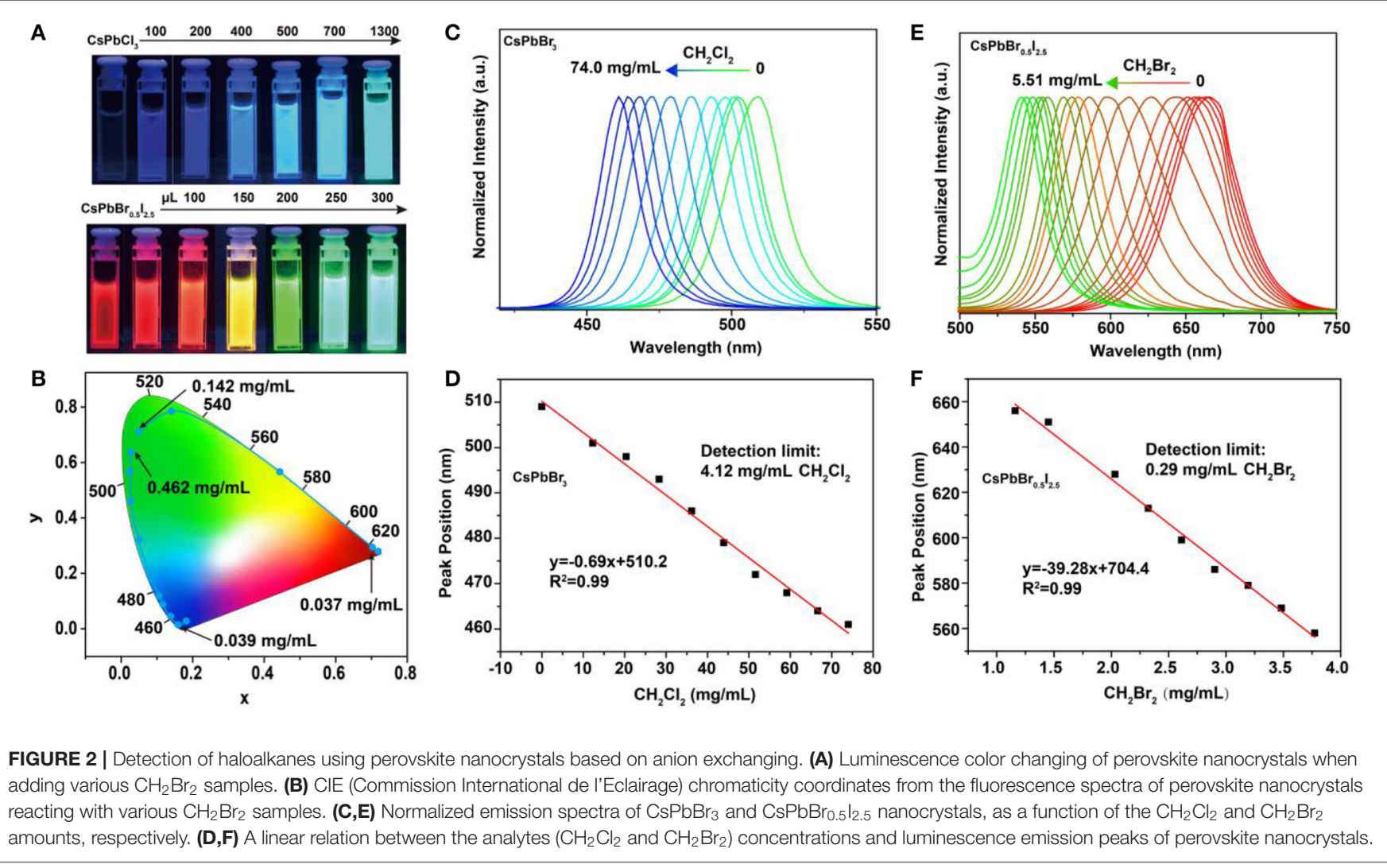


method was sufficient for qualitative and quantitative analysis of halide compounds.

We carried out experiments to fabricate a paper-based microfluidic device for achieving on-site detection of haloalkane. Figure 3a shows the design of a six-channel paper microchip containing three types of perovskite nanocrystals. The detection of the targeted haloalkane samples was implemented by diffusing them into the perovskite nanocrystals via anion exchangemediated reaction. In a typical experiment, we deposited the perovskite nanocrystals of $\mathrm{CsPbCl}_{3}, \mathrm{CsPbBr}_{3}$, and $\mathrm{CsPbBr}{ }_{0.5} \mathrm{I}_{2.5}$ in the microchannels which emitted deep blue, green, and red fluorescence under a 365-nm excitation (Figure 3b). The $\mathrm{CH}_{2} \mathrm{Cl}_{2}$ sample was added to the central region of the designed paper microfluidic device. Our experiments indicated that the green fluorescence of $\mathrm{Cs} \mathrm{PbBr}_{3}$ nanocrystals was quickly changed into blue as a result of anion exchanging-mediated reaction under UV illumination. This suggested that our design is suitable for a fast detection of $\mathrm{CH}_{2} \mathrm{Cl}_{2}$ sample by convenient colorimetric sensing (Figure 3c). In a parallel set of experiments, we demonstrated that the device can also be employed to qualitatively detect $\mathrm{CH}_{2} \mathrm{Br}_{2}$ sample through monitoring the fluorescence color change of $\mathrm{CsPCl}_{3}$ and $\mathrm{CsPbrr}_{0.5} \mathrm{I}_{2.5}$ channels
(Figure 3d). We further demonstrated that our method is suitable for the detection of a mixed sample containing both $\mathrm{CH}_{2} \mathrm{Cl}_{2}$ and $\mathrm{CH}_{2} \mathrm{Br}_{2}$ (Figure 3e; Supplementary Figure 7). The reaction mechanism for the haloalkane sensing is illustrated in Figure 3f, an anion exchange process with $\mathrm{CsPbX}_{3}(\mathrm{X}=\mathrm{Cl}, \mathrm{Br}$, or I) nanocrystals. Haloalkane molecules, such as $\mathrm{CH}_{2} \mathrm{Br}_{2}$, were introduced in-situ near the surface of perovskite nanocrystals to implement the anion-exchange reaction, upon either an addition of nucleophilic trialkyl phosphines (TOP) or a UV-photoninduced electron transfer from $\mathrm{CsPbX}_{3}$ nanocrystals (Figure 3f). The change of halide composition after the anion exchanging leads to continuous changes of the bandgap, as well as the absorption and emission spectra in the perovskite nanocrystals (Figure 3g).

The physical process for haloalkane detection was investigated by monitoring the chemical kinetics of the anion-exchanged reaction between perovskite nanocrystals and haloalkane. A solvent mixture of $\mathrm{CH}_{2} \mathrm{Br}_{2}$ and cyclohexane containing nucleophilic TOP was homogeneously mixed with $\mathrm{CsPbBr}_{0.5} \mathrm{I}_{2.5}$ nanocrystals. Under UV illumination, we observed a gradual change in the photoluminescence emission color, as a function of halide exchanging time. Figure $4 \mathrm{~A}$ shows the green
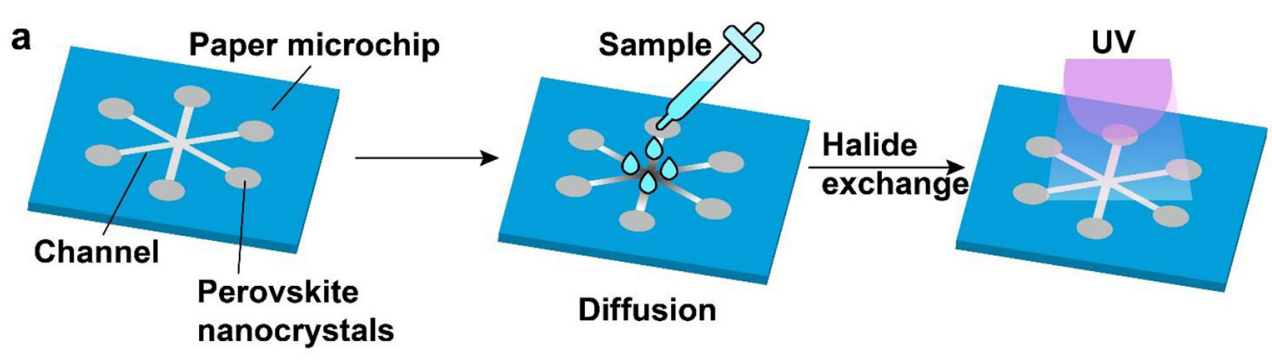

Diffusion
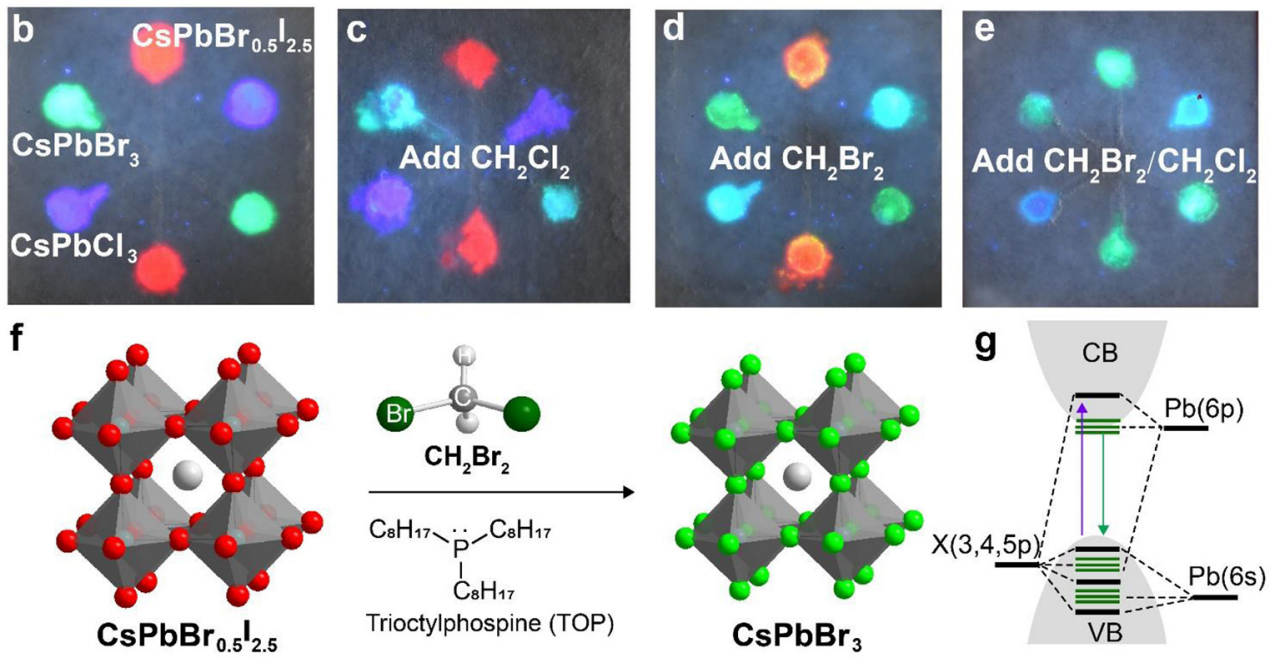

FIGURE 3 | Schematic of a perovskite microfluidic platform for on-site detection of haloalkane. (a) Design of a paper-based microfluidic chip for multichannel detection of haloalkane. The perovskite nanocrystals were firstly filled in the paper microchip, and then the analyte samples were dropped and diffused along the microchannel. Upon UV irradiation, on-site sensing can be implemented as a result of a fast anion exchange reaction. (b) Luminescence image of a paper-based microfluidic device filled with perovskite nanocrystals ( $\mathrm{CsPbCl}_{3}, \mathrm{CsPbBr}_{3}$, and $\mathrm{CsPbBr}_{0.5} \mathrm{I}_{2.5}$ ). The photograph was taken under a 365-nm UV excitation. (c-e) Colorimetric sensing of $\mathrm{CH}_{2} \mathrm{Cl}_{2}$ and $\mathrm{CH}_{2} \mathrm{Br}_{2}$, using the paper-based microfluidic device from (b). The photographs were taken under a 365-nm UV excitation. (f) Reaction mechanism of a nucleophile (TOP)-mediated anion exchange between perovskite nanocrystals and halides. (g) Schematic of the electronic structures for perovskite nanocrystals. 


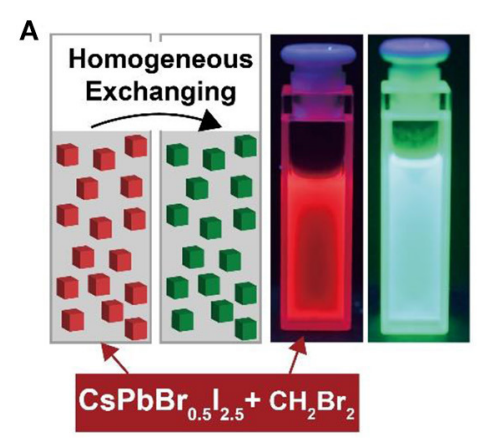

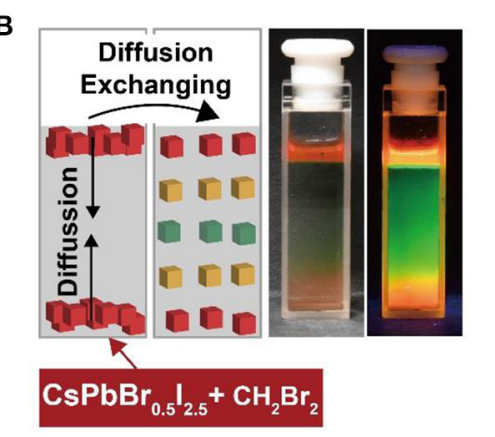

C

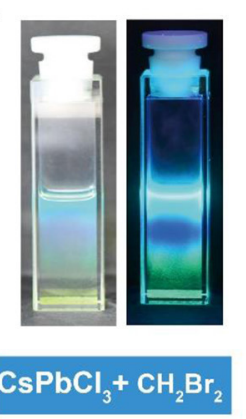

FIGURE 4 | Direct observation of chemical kinetics of anion exchanging between perovskite nanocrystals and haloalkanes through slow solvent diffusion. (A) Homogeneous anion exchanging between $\mathrm{CsPbBr} .5 \mathrm{I}_{2.5}$ and $\mathrm{CH}_{2} \mathrm{Br}_{2}$. The luminescent images were recorded before and after anion exchanging under a 365 -nm $\mathrm{UV}$ illumination. (B) Diffusion anion exchanging between $\mathrm{CsPbBr}_{0.5} \mathrm{I}_{2.5}$ and $\mathrm{CH}_{2} \mathrm{Br}_{2}$. (C) Diffusion anion exchanging between $\mathrm{CsPbCl}_{3}$ and $\mathrm{CH}_{2} \mathrm{Br}_{2}$.

photoluminescence emission via anion exchange for $1 \mathrm{~min}$. In a parallel experiment, $10 \mu \mathrm{L}$ of $\mathrm{CsPbBr}_{0.5} \mathrm{I}_{2.5}$ perovskite nanocrystals were dropped into a mixture of $\mathrm{CH}_{2} \mathrm{Br}_{2}$ and cyclohexane containing nucleophilic TOP. Intriguingly, a colorful rainbow-based photoluminescence emission was observed under UV illumination (Figure 4B). The gradual transition in photoluminescence emission color from red to green was attributed to the slow diffusion of perovskite nanocrystals into the solvent. A similar result was also obtained by diffusing $\mathrm{CsPCl}_{3}$ nanocrystals into the mixture of $\mathrm{CH}_{2} \mathrm{Br}_{2}$ and cyclohexane (Figure 4C; Supplementary Figure 8). The direct experimental observation on the chemical kinetics of anion exchange in perovskite nanocrystals offers a powerful strategy for in-depth understanding of the physical process of anion exchanging in perovskite nanocrystals.

In summary, we have developed a perovskite-based paper microfluidic sensor for detection of haloalkanes through anion exchanging. Our experimental results demonstrated that, by combing with perovskite nanocrystals, the paperbased multichannel microfluidic device offers a low-cost, highthroughput and convenient platform for fast colorimetric sensing of haloalkanes. The direct experimental observation on chemical kinetics of slow diffusion-mediated anion exchanging in perovskite nanocrystals may be valuable for a fundamental understanding on the materials synthesis and optical properties of perovskite nanocrystals for various applications, such as Xray imaging and photocatalysis (Chen et al., 2018; Zhu X. et al., 2019; Ou et al., 2021). Future work can be devoted to designing the flexible microfluidic sensors suitable for achieving on-site qualitative and quantitative analysis of haloalkane and for both visual and instrumental readout.

\section{REFERENCES}

Akkerman, Q. A., Raino, G., Kovalenko, M. V., and Manna, L. (2018). Genesis, challenges, and opportunities for colloidal lead halide perovskite nanocrystals. Nat. Mater. 17, 394-405. doi: 10.1038/s41563-0180018-4

\section{DATA AVAILABILITY STATEMENT}

The original contributions presented in the study are included in the article/Supplementary Material, further inquiries can be directed to the corresponding authors.

\section{AUTHOR CONTRIBUTIONS}

LX, ZY, CL, QC, and HY contributed to the conception and design of the experiments. LX, JZ, and ZY contributed to the materials synthesis, sample testing, and wrote the draft of the manuscript. QW, XC, and $\mathrm{XO}$ performed the data analysis. CL, QC, and $\mathrm{HY}$ wrote the manuscript. All authors contributed to approving the submitted version.

\section{FUNDING}

This work was supported by the National Key R\&D Program of China (2020YFA0709903, 2020YFA0210800), the National Natural Science Foundation of China (21635002), the Academic Research Fund of Fuzhou University (0041-510860), the Joint Funds for the innovation of science and Technology of Fujian province (2018Y9024), and the Natural Science Foundation of Fujian Province (2018J01687).

\section{SUPPLEMENTARY MATERIAL}

The Supplementary Material for this article can be found online at: https://www.frontiersin.org/articles/10.3389/fchem. 2021.682006/full\#supplementary-material

Cate, D. M., Adkins, J. A., Mettakoonpitak, J., and Henry, C. S. (2015). Recent developments in paper-based microfluidic devices. Anal. Chem. 87, 19-41. doi: 10.1021/ac503968p

Chen, Q., Wu, J., Ou, X., Huang, B., Almutlaq, J., Zhumekenov, A. A., et al. (2018). All-inorganic perovskite nanocrystal scintillators. Nature 561, 88-93. doi: 10.1038/s41586-018-0451-1 
Daud, N. H., Oslan,S. N., Leow, T. C., and Salleh, A. B (2018). Development of an enzyme-based fiber optic biosensor for detection of haloalkanes. Sens. Rev. 39, 810-818. doi: 10.1108/SR-01-2019-0001

Fu, Q., Fujii, M., and Kwon, E. (2020). Development and application of a high-precision algorithm for nontarget identification of organohalogens based on ultrahigh-resolution mass spectrometry. Anal. Chem. 92, 13989-13996. doi: 10.1021/acs.analchem.0c02899

Geng, C., Xu, S., Zhong, H., Rogach, A. L., and Bi, W. (2018). Aqueous synthesis of methylammonium lead halide perovskite nanocrystals. Angew. Chem. Int. Ed. 57, 9650-9654. doi: 10.1002/anie.201802670

Gul, I., Wang, Q., Jiang, Q., Fang, R., and Tang, L. (2020). Enzyme immobilization on glass fiber membrane for detection of halogenated compounds. Anal. Biochem. 609:113971. doi: 10.1016/j.ab.2020.113971

Huang, S., Li, Z., Kong, L., Zhu, N., Shan, A., and Li, L. (2016). Enhancing the stability of $\mathrm{CH}_{3} \mathrm{NH}_{3} \mathrm{PbBr}_{3}$ quantum dots by embedding in silica spheres derived from tetramethyl orthosilicate in "waterless" toluene. J. Am. Chem. Soc. 138, 5749-5752. doi: 10.1021/jacs.5b13101

Kinani, A., Kinani, S., and Bouchonnet, S. (2016). Formation and determination of organohalogen by-products in water. Part III. Characterization and quantitative approaches. Trends Anal. Chem. 95, 295-305. doi: 10.1016/j.trac.2016.09.013

Kovalenko, M. V., Protesescu, L., and Bodnarchuk, M. I. (2017). Properties and potential optoelectronic applications of lead halide perovskite nanocrystals. Science 358, 745-750. doi: 10.1126/science.aam7093

Leri, A. C., Hay, M. B., Lanzirotti, A., Rao, W., and Myneni, S. C. (2006). Quantitative determination of absolute organohalogen concentrations in environmental samples by X-ray absorption spectroscopy. Anal. Chem. 78, 5711-5718. doi: 10.1021/ac060476m

Li, B., Cui, L., and Li, C. (2020). Macrocycle Co-crystals showing vapochromism to haloalkanes. Angew. Chem. Inter. Ed. 59, 22012-22016. doi: 10.1002/anie.202010802

Li, F., Feng, Y., Huang, Y., Yao, Q., Huang, G., Zhu, Y., et al. (2021). Colorimetric sensing of chloride in sweat based on fluorescence wavelength shift via halide exchange of $\mathrm{CsPbBr}_{3}$ perovskite nanocrystals. Mikrochim. Acta 188:2. doi: 10.1007/s00604-020-04653-5

Li, Z., Kong, L., Huang, S., and Li, L. (2017). Highly luminescent and ultrastable $\mathrm{Cs} \mathrm{PbBr} 3$ perovskite quantum dots incorporated into a silica/alumina monolith. Angew. Chem. 129, 8246-8250. doi: 10.1002/ange.201703264

Miller, B. S., Bezinge, L., Gliddon, H. D., Huang, D., Dold, G., Gray, E. R., et al. (2020). Spin-enhanced nanodiamond biosensing for ultrasensitive diagnostics. Nature 587, 588-593. doi: 10.1038/s41586-020-2917-1

Ou, X., Qin, X., Huang, B., Zan, J., Wu, Q., Hong, Z., et al. (2021). Highresolution X-ray luminescence extension imaging. Nature 590, 410-415. doi: 10.1038/s41586-021-03251-6

Pan, D., Fu, Y., Chen, J., Czech, K. J., Wright, J. C., and Jin, S. (2018). Visualization and studies of ion-diffusion kinetics in cesium lead bromide perovskite nanowires. Nano Lett. 18, 1807-1813. doi: 10.1021/acs.nanolett.7b05023

Parobek, D., Dong, Y., Qiao, T., Rossi, D., and Son, D. H. (2017). Photoinduced anion exchange in cesium lead halide perovskite nanocrystals. J. Am. Chem. Soc. 139, 4358-4361. doi: 10.1021/jacs.7b01480

Protesescu, L., Yakunin, S., Bodnarchuk, M. I., Krieg, F., Caputo, R., Hendon, C. H., et al. (2015). Nanocrystals of cesium lead halide perovskites $(\operatorname{CsPbX}(3), X$
$=\mathrm{Cl}, \mathrm{Br}$, and I): novel optoelectronic materials showing bright emission with wide color gamut. Nano Lett. 15, 3692-3696. doi: 10.1021/nl5048779

Roveretto, M., Li, M., Hayeck, N., Brüggemann, M., Emmelin, C., Perrier, S., et al. (2019). Real-time detection of gas-phase organohalogens from aqueous photochemistry using orbitrap mass spectrometry. ACS Earth Space Chem. 3, 329-334. doi: 10.1021/acsearthspacechem.8b00209

Wong, Y., Wu, W., Wang, T., Ng, J. D. A., Khoo, K. H., Wu, J., et al (2019). Color patterning of luminescent perovskites via light-mediated halide exchange with haloalkanes. Adv. Mater. 31:e1901247. doi: 10.1002/adma.201 901247

Xie, L., Zi, X., Zeng, H., Sun, J., Xu, L., and Chen, S. (2019). Low-cost fabrication of a paper-based microfluidic using a folded pattern paper. Anal. Chim. Acta 1053, 131-138. doi: 10.1016/j.aca.2018.12.001

Xing, G., Mathews, N., Lim, S. S., Yantara, N., Liu, X., Sabba, D., et al. (2014). Lowtemperature solution-processed wavelength-tunable perovskites for lasing. Nat. Mater. 13, 476-480. doi: 10.1038/nmat3911

Yin, J., Maity, P., Bastiani,M. D., Dursun, I., Bakr, O. M., Bredas, J. L., et al. (2017). Molecular behavior of zero-dimensional perovskites. Sci. Adv. 3:e1701793. doi: 10.1126/sciadv.1701793

Yin, W., Li, H., Chesman, A. S. R., Tadgell, B., Scully, A. D., Wang, M., et al. (2021). Detection of halomethanes using cesium lead halide perovskite nanocrystals. ACS Nano 15, 1454-1464. doi: 10.1021/acsnano.0c08794

Yoon, Y. J., Lee, K. T., Lee, T. K., Kim, S. H., Shin, Y. S., Walker, B., et al. (2018). Reversible, full-color luminescence by post-treatment of perovskite nanocrystals. Joule 2, 1-12. doi: 10.1016/j.joule.2018.07.012

Zheng, W., Huang, P., Gong, Z., Tu, D., Xu, J., Zou, Q., et al. (2018). Near-infrared-triggered photon upconversion tuning in all-inorganic cesium lead halide perovskite quantum dots. Nat. Commun. 9:3462. doi: 10.1038/s41467-018-05947-2

Zhou, Q., Bai, Z., Lu, W., Wang, Y., Zou, B., and Zhong, H. (2016). In situ fabrication of halide perovskite nanocrystal-embedded polymer composite films with enhanced photoluminescence for display backlights. Adv. Mater. 28, 9163-9168. doi: 10.1002/adma.201602651

Zhu, X., Lin, Y., Martin, J. S., Sun, Y., Zhu, D., and Yan, Y. (2019). Lead halide perovskites for photocatalytic organic synthesis. Nat. Commun. 10:2843. doi: 10.1038/s41467-019-10634-X

Zhu, Y., Li, F., Huang, Y., Lin, F., and Chen, X. (2019). Wavelengthshift-based colorimetric sensing for peroxide number of edible oil using $\mathrm{CsPbBr}_{3}$ perovskite nanocrystals. Anal. Chem. 91, 14183-14187. doi: 10.1021/acs.analchem.9b03267

Conflict of Interest: The authors declare that the research was conducted in the absence of any commercial or financial relationships that could be construed as a potential conflict of interest.

Copyright (c) 2021 Xie, Zan, Yang, Wu, Chen, Ou, Lin, Chen and Yang. This is an open-access article distributed under the terms of the Creative Commons Attribution License (CC BY). The use, distribution or reproduction in other forums is permitted, provided the original author(s) and the copyright owner(s) are credited and that the original publication in this journal is cited, in accordance with accepted academic practice. No use, distribution or reproduction is permitted which does not comply with these terms. 